\title{
Phantom limb sensations in the ear of a patient with a brachial plexus lesion
}

Mariella Pazzaglia ${ }^{1,2}$, Giulia Galli ${ }^{2 *}$, Giuliana Lucci $^{1,2^{*}}$, Giorgio Scivoletto ${ }^{2}$, Marco Molinari ${ }^{2}$, Patrick Haggard ${ }^{3}$.

${ }^{1}$ Department of Psychology, University of Rome "La Sapienza," Via dei Marsi 78, 00185 Rome, Italy

2 IRCCS Santa Lucia Foundation, Via Ardeatina 306, 00100 Rome, Italy

${ }^{3}$ Institute of Cognitive Neuroscience, University College London, London, UK

* indicates shared authorship

Correspondence to:

Mariella Pazzaglia

Dipartimento di Psicologia, Università di Roma "La Sapienza,"

Via dei Marsi 78, 00185, Roma, Italia.

Email: mariella.pazzaglia@uniroma1.it

- Keywords: phantom sensation, ear, brain plasticity, ear hand remapping, brachial plexus avulsion

Running title: Ear Phantom Sensations

Contributors: MP conducted the first neuropsychological evaluation on patient, study concept and design, acquisition of data, analysis and interpretation, writing and drafting of the manuscript, critical revision of the manuscript for important intellectual content. GG performed acquisition, analysis and interpretation of data, drafting of the manuscript, critical revision of the manuscript for important intellectual content. GG and MM performed acquisition of neurologic data. GL: performed the acquisition, analysis, interpretation and writing of EEG data. PH: analysis and interpretation of data, drafting of the manuscript, critical revision of the manuscript for important intellectual content. All authors read and approved the final manuscript.

\section{Summary}

Referred phantom sensations are frequently reported following a peripheral injury. However, very few cases describe such sensations of the ear, and it remains unclear how the aural nerve territory can be remapped to one specific peripheral nerve region. We report on a patient with brachial plexus avulsion who underwent sensory testing and was asked to report the location of the 
stimulated site and any other sensations experienced. The patient spontaneously described the sensation of his arm being separate from his body. Despite visual input, he felt that his fist was closed, with his thumb pointing inward. Importantly, he felt clear and reproducible sensations from the affected arm when the ipsilateral ear was touched. These referred sensations were noted just 15 days after sustaining the injury. The arm nerve territory was systematically remapped to a specific aural nerve territory by applying both manual and electrical stimulation. Stimulation of the external ear, which is innervated by the vagus nerve, showed high spatial specificity for the dorsal and volar skin surfaces of the limb, and clearly delineated digits. Somatosensory-evoked potentials indicated that cortical adaptation in the somatosensory stream transferred a spatially organized map of the limb to the skin of the outer ear. This referral of sensations to the ear, as distinct from the face, provides evidence of highly specific topographical reorganization of the central nervous system following peripheral injury. Rapid map changes in the phantom sensation to the ear as a function of stimulation of vagus nerve suggest that the reorganization process can occur in cortex rather than in the brainstem.

\section{Introduction}

With the phantom limb phenomenon, one experiences the vivid sensation that a missing limb is still attached to the body and is moving appropriately with other body parts (Ramachandran \& Hirstein, 1998). These sensations, which are not necessarily painful, typically emerge immediately after an injury, are topographically precise, and vanish with time (Flor, Nikolajsen, \& Staehelin Jensen, 2006). Several clinical studies have reported sensations from the missing arm, delivered from sensory stimuli applied to the ipsilateral face (Halligan, Marshall, \& Wade, 1994; Ichinose et al., 2017; Montoya et al., 1998; Ramachandran \& Hirstein, 1998; Ramachandran \& Rogers-Ramachandran, 2000). More than $45 \%$ of amputees experience this hand-to-face remapping (Collins et al., 2017)_even many years after the amputation, as in the Ramachandran patient (Ramachandran \& Hirstein, 1998). Using Penfield's topographical cortical representation of the body (Penfield \& Rasmussen, 1950), most hypotheses are based on the proximity of adjacent brain areas, such as the face and the remaining intact limb areas. The changes in the somatotopic organization are considered manifestations of cortical reorganization. The hand topography, previously assigned to processing the now-silenced sensory information, is invaded by new input from the face.

Accordingly, cortical map studies report that in individuals with phantom limb sensation, facial stimulation can functionally reactivate the zone of the primary somatosensory cortex that represents the hand (Birbaumer et al., 1997; Flor et al., 1995; Lotze, Flor, Grodd, Larbig, \& 
Birbaumer, 2001). A complementary pattern also occurs, wherein tactile stimulation of the human hand can be traced to the cortical representation of the face, following the application of botulin toxin in the treatment of wrinkles (Haenzi, Stefanics, Lanaras, Calcagni, \& Ghosh, 2014). However, recent evidence indicates that these somatosensory cortex shifts in the missing hand territory are smaller than initially suggested (Makin \& Bensmaia, 2017). The original representation of the missing hand's individual fingers persists in the primary somatosensory cortex, even decades after deafferentation (Makin \& Bensmaia, 2017; Makin et al., 2013; Makin, Scholz, Henderson Slater, Johansen-Berg, \& Tracey, 2015). Accordingly, functional changes observed in the somatosensory cortex following peripheral input loss, could be attributed to reorganization in subcortical areas in the afferent pathway (Chand \& Jain, 2015; Kambi et al., 2014), which could potentially contribute to changes in the somatosensory cortex. In particular, after limb denervation there could be new connections between the limb territories in the brainstem and the trigeminal afferent system, which receives signals from the face. In other words, the reorganization of the somatosensory cortex seems to reflect to the reorganization of ascending somatosensory pathways, rather than reorganization of intrinsic maps within the somatosensory cortex.

The referral of phantom sensations elicited by the stimulation of body sites adjacent to the affected limb is reported in 60-95\% of individuals with amputated limbs (Melzack, 1990; Weeks \& Tsao, 2010), but the phenomenon is also frequently reported in cases of an intact limb with a loss of sensory and motor innervation due to nerve avulsion (Sherman, Griffin, Evans, \& Grana, 1992), spinal cord injury (Cook \& Druckemiller, 1952), or brachial plexus lesions (Finnerup et al., 2010). Unlike phantom limb phenomena caused by amputation, the precise incidence and natural course of phantom sensations in brachial plexus avulsion are largely unknown (Son \& Ha, 2015). Brachial plexus injury (BPI) is a type of peripheral nerve injury that occurs following the avulsion of all the lower roots (C5-T1) of a limb that is anatomically present. In BPI, unlike amputation, the physical body remains unaltered, but there is a complete loss of motor and sensory function in the affected limb. From the brain's viewpoint, BPI and amputation could be quite similar: in both cases, the absence of sensory input from a certain body part leads to the absence of a corresponding representation in the brain. Research findings suggest that phantom reduplication in BPI depends on the presence of rapid somatosensory reorganization and compensatory mechanisms (Bhat, Indira Devi, Bharti, \& Panda, 2017)— presumably the same mechanisms that are responsible for phantom sensations in amputees. In BPI, phantom limbs are usually experienced without proprioceptive or sensory qualities (Hunter, Katz, \& Davis, 2003), or when assuming a distorted posture (Tsao, Finn, \& Miller, 
2016), and with sensation transferred to the face (Tsao et al., 2016). Generally, the phantoms are perceived as "normal" in size and shape, and are only rarely experienced as being shortened or telescoped (Katz, 1992; Parry, 1980). In approximately one-half of all cases, the phenomenon is pain-free; in the other cases, pain may be severe and long-lasting (Shankar, Hansen, \& Thomas, 2015). To shed further light on these phenomena, in this study, we describe phantom sensations in a patient with an intact but denervated limb, following brachial plexus avulsion.

\section{Materials and Methods}

\subsection{Case description}

A.M. (male; right-handed; 25 years; 13 years of education) was diagnosed with a brachial plexus lesion based on clinical examination (continued paralysis/paresis and sensory disturbance, muscle atrophy, decreased tendon reflexes, and Horner's syndrome), conventional magnetic resonance imaging (MRI) scans (Fig. 1), and electromyogram (EMG) documenting the complete absence of the evoked compound motor action potential and sensory action potential on the left ulnar, median, and radial nerves.

The patient was examined in the post-acute phase, 15 days after the injury occurred. The patient did not suffer a head injury and had no signs of psychiatric disorder or a history of substance abuse. Informed written consent was obtained for all procedures, and the local ethics committee of Santa Lucia Foundation approved the study.
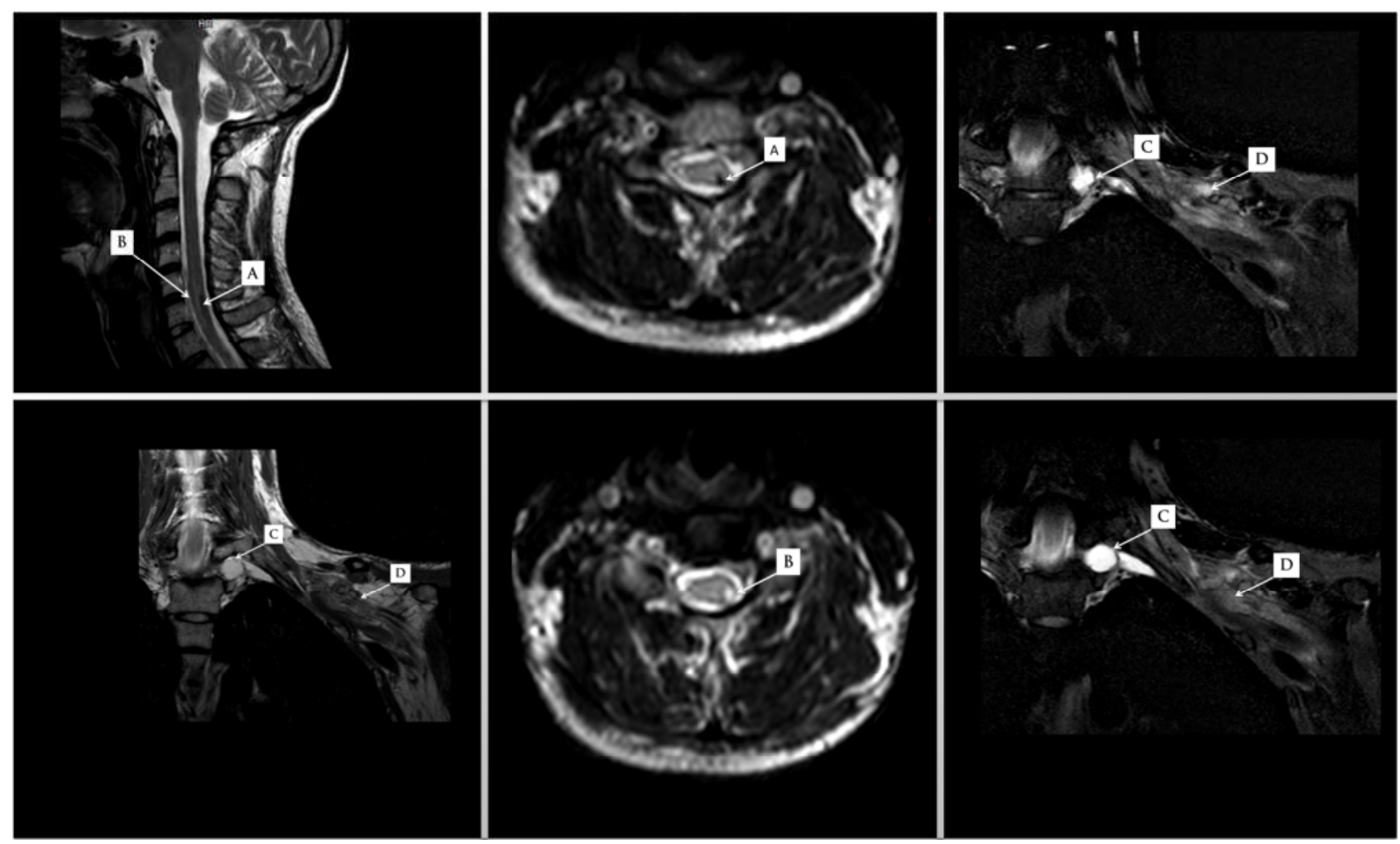
Clinical examinations were performed to investigate sensory loss by administering sensory stimuli in a random order to the face and limbs with eyes closed. Sensory loss was tested with SemmesWeinstein Monofilaments (Touch-TestSensory Evaluators; Stoelting Co., Wood Dale, IL, USA) in sensory dermatomes of the ipsi- (left) and contralesional (right) sides. Before testing, each stimulated point was marked to ensure that the same points of both upper limbs were tested across trials. To measure the two-point discrimination thresholds, we used the method of limits with interleaved ascending (initial distance of $0.2 \mathrm{~cm}$ ) and descending staircases (initial distance was the maximal achievable for the explored dermatomes). Threshold measurements were administrated to each point on the healthy limb using monofilaments mounted on an electronic vernier caliper until reaching the minimal distance at which the stimuli were correctly discriminated for three consecutive stimulations. Monofilaments were calibrated for $4 \mathrm{~g}$, which was clearly above detection threshold for all parts of the healthy right limb. After the estimate of threshold, 25 predefined different trials (10 double, 10 single, 5 no touch) were randomly administered on each dermatome, according to the protocol shown in Fig. 2. The patient was asked to verbally report whether he felt zero, one, or two separate points.

To test for the presence and distribution of residual sensations and referred sensations, A.M. was also gently touched with a Q-tip on various parts of his body (for example face, ears, neck, and legs). He was instructed to report what he felt and exactly where he felt it. For sites where touch evoked a referred sensation on the limb, following standard procedure, we also investigated effects of distinct categories of stimulation: digital pressure, no stimulation, tapping, shaking, and gentle stroking in a distal to proximal direction and vice versa, with approximately a 3 -cm stroke lasting 1 s.

\subsection{Somatosensory evoked potentials acquisition}

A continuous electroencephalogram (EEG) was recorded using BrainVision ${ }^{\mathrm{TM}}$ Recorder System (BrainProducts $\mathrm{GmbH}$, Munich, Germany) with 64 electrodes mounted according to the 10-10 International System and referenced to $\mathrm{Cz}$ electrode. The electrode impedances were kept $<5 \mathrm{k} \Omega$. All signals were digitized at $5000 \mathrm{~Hz}$ and stored on a disk for offline averaging. The EOG was also recorded by electrodes at right external canthi and below the left eye.

The somatosensory evoked potentials (SEPs) were obtained by electrical stimulation of both left (where touch evoked a referred sensation on the limb) and right (control site) ears, using a pair of silver ball electrodes (i.e., putting the anode $(+)$ on the ear and the cathode (-) on the Scapha [Fig. 5]). The electrical stimulus was a constant voltage square pulse wave. We used short stimulus 
duration of $0.08 \mathrm{~ms}$, to minimize electrical artifacts for SEPs. The stimulus intensity was set to produce subjective sensation also on the patient's elbow, i.e., at $8 \mathrm{~mA}$. To prevent habituation, the ISI was set randomly between 350 and $650 \mathrm{~ms}$. A total of 1000 stimuli per ear were delivered.

Data analysis was performed using the Brain Vision Analyzer 2 software package. The EEG was segmented for each electrical stimulus, producing epochs of $300 \mathrm{~ms}$ ( -50 to $250 \mathrm{~ms}$ ). Data points affected by electrical stimulation artifacts (from -10 to $10 \mathrm{~ms}$ with respect to the stimulus onset) were removed using linear interpolation. Eye blinks, saccades, and pulse artifacts were corrected using an independent component analysis (ICA). A DC detrend was applied using the pre- and poststimulus period as the baseline. For the artifact rejection, a manual selection procedure was adopted by rejecting trials that were still contaminated by artifacts like ocular movements and muscular contractions. The averages were filtered using an IIR filter (0.01-100 Hz, at $12 \mathrm{db} / \mathrm{oct}$ each). Baseline correction was conducted using the interval from $-50 \mathrm{~ms}$ to $0 \mathrm{~ms}$ as the pre-stimulus baseline.

\section{Behavioral Results}

On questioning, A.M. reported the distinctive experience of his left arm being detached and external to him, as if it had been artificially attached to the side of his body. He reported feeling that his left hand was closed in a fist, with the thumb flexed and pointing inwards. In reality, the arm was supported and held in a flexed position by a sling, and the hand and fingers were fully relaxed and in extension. Thus A.M. reported a "felt" experience of his arm that was quite different from the real and seen posture of the arm. He also experienced unintentional attempts to move. He reported occasionally feelings of constricting, severe, and sharp pain, which started gradually from his shoulder and progressed to his fingertips. Interestingly, the investigating neurologist ascribed these painful sensations to the initial trauma, rather than to phantom pain.

\subsection{Tactile sensitivity}

The patient's results are shown in Fig. 2. On physical examination, A.M. had a perfect performance for the two-point discrimination task, and for single and no touch stimuli, for all tested dermatomes on the right (contralesional) side, demonstrated a normal and correct sensitivity in the spared hemisoma.

On the left (ipsilesional) side, for tactile spatial discrimination, A.M. was completely unable to report whether the touch was applied to one or two points simultaneously, even at very high spatial separations. Regarding tactile detection, A.M. was able to detect that he had been touched on $\mathrm{T}_{1}$ dermatome, with a performance clearly above chance. However, he was unable to detect that he had 
been touched on $\mathrm{C}_{6}, \mathrm{C}_{7}$, and $\mathrm{C}_{8}$ dermatomes, where his performance was at chance levels. These results suggest impairment of fine touch discrimination on left arm, together with a partial preservation of tactile detection.

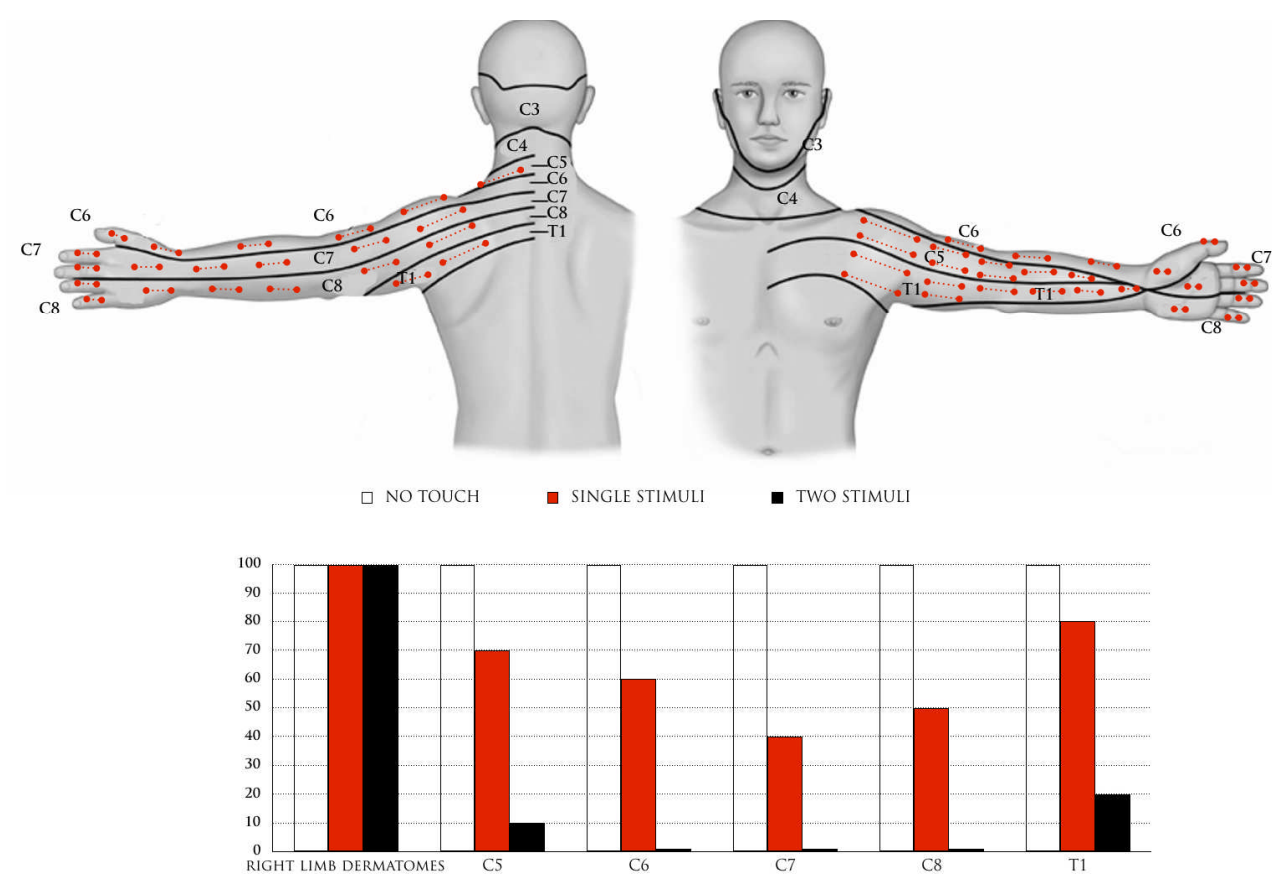

\subsubsection{An entire map of the missing limb on ear}

A.M.'s responses to touch were all correct for stimulation on the right side of the body. He also performed at $100 \%$ on the left side of the face, until the left ear was stimulated, initially by accident. At this point, he spontaneously expressed surprise and concern, and reported feeling the "presence" of the injured arm. We therefore applied systematic sensory stimuli to the ipsi- and contralateral ears to investigate the topography of sensations arising from the injured arm. No limb sensations occurred when we stimulated the right ear. Conversely, touches on the left ear were able to elicit clear and specific touch sensations arising from the dorsal and ventral sides of the arm, hand, and individual fingers of the affected left hand (see Fig. 3). Since four separate sensory nerves supply the skin of the ear ([1] greater auricular nerve, [2] auriculotemporal nerve, [3] lesser occipital nerve, and [4] auricular branch of the vagus nerve [Fig. 3]), we plotted these referred sensations on a map of the relevant nerve territories. Specifically, touching the anterior lobule of the ear, which is innervated by the greater auricular nerve (area 1), did not induce any specific sensation on the arm; rather, A.M. experienced generalized tingling on the shoulder. Touching the curling rim of the most lateral border of the auricle, which is innervated by the auriculotemporal nerve (area 2), led to sensations arising from the external surface of the left arm, extending from the shoulder to the elbow. Touching the lower portion of the helix, which is innervated by the lesser occipital nerve 
(area 3), led to sensations referred to the area between the left elbow and wrist and elicited vague perceptions referred to the hand's dorsal surface. Stimulation of central internal portion of the ear between the cymba and cavum of concha, which is innervated by the vague nerve (area 4), yielded sensation arising from the dorsal surface of the left hand, and, in a clearly delineated fashion, on the digits of the left hand, extending from the little finger to thumb.

Importantly, stimulation of the posterior portion of the ear led to referred sensations arising from the internal surface of the left arm between the shoulder to elbow, while more internal stimulation led to referred sensations on the individual digits of the left hand. Again, these referred sensations showed a clearly delineated pattern extending from the index finger to the thumb, but in this case on the palm of his left hand. Therefore, the hand nerve territory was systematically remapped, preserving delineation between the dorsal and ventral arm, and between individual digits.

Different patterns of stroking movement delivered to the helix of ear (e.g., continuous stroking versus single tapping versus shaking) were felt as the same sensation on the left limb.

The spatial direction of stroking movements on the helix (from the top to the lobule) produced a sensation of something moving along his arm coherently (from the shoulder to the hand). Different directions of stroking the ear were systematically associated with different experiences of directed movement across the skin of the left arm. Interestingly, when asked to report the pleasantness or unpleasantness of the referred sensations, A.M. rated them as pleasant (8 on a 10-point visual analogue scale [VAS] where 0 and 10 are "very unpleasant" and "very pleasant," respectively).

Finally, touching the ear produced changes in the experienced posture of the left arm. With his eyes closed, A.M. indicated with his healthy arm that the touch on his left ear led to the experience of the left arm no longer being bent, but instead being outstretched and normally attached to his body. 


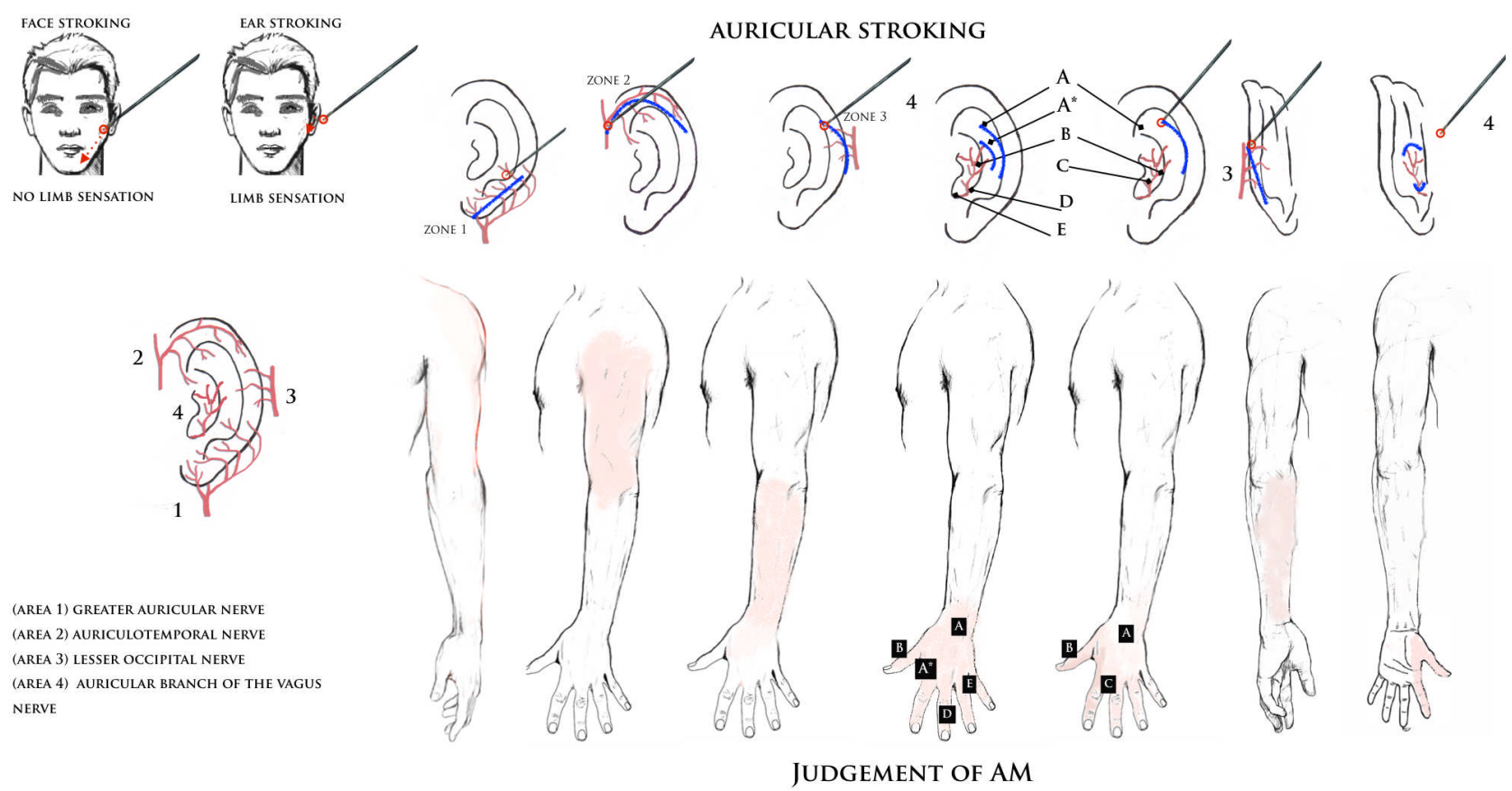

We repeated some of these procedures using non-noxious electrical stimulation (STM stimulator 140, High Technology Laboratory) to quantify the evoked limb sensations. Stimulation intensities ranged from 4 to $9 \mathrm{~mA}$ (duration of $0.05 \mathrm{~ms}$ ), defined before starting the experimental session; stimuli were not painful but clearly perceived above threshold. One point on the face and three points on the left and right ear (skin innervated from auriculotemporal nerve, lesser occipital nerve, and auricular branch of the vagus nerve) were stimulated (see Fig.4). We recorded 12 sessions in random order.

The patient's responses to electrical stimulation were precise and consistent with the mapping identified by manual stimulation. Phantom limb sensation intensity was rated as 8 on a 10-point VAS scale ( $0=$ "no sensation," $10=$ "normal sensation"). "Normal" was defined as the sensations evoked by electrical stimulation of the right ear, and at the corresponding points on the right arm. Interestingly, with increasing intensity of stimulation applied to the ear, he reported a concomitant increase in the strength of the sensation at the corresponding referral point on the limb map. No phantom sensations were reported for same stimulation of the face. 


\section{ELECTRICAL STIMULATION}

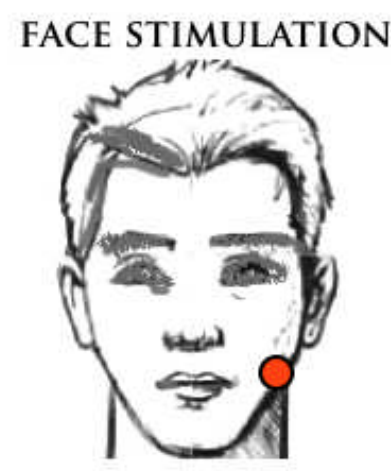

NO LIMB SENSATION

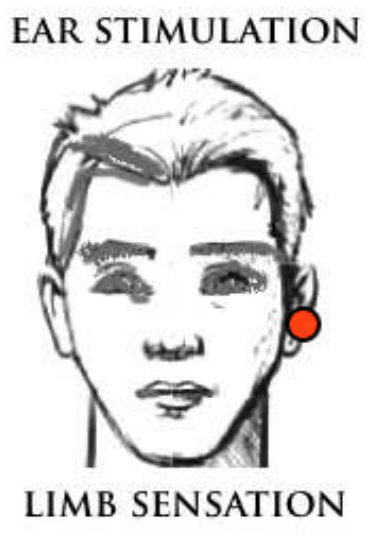

JUDGEMENT OF AM
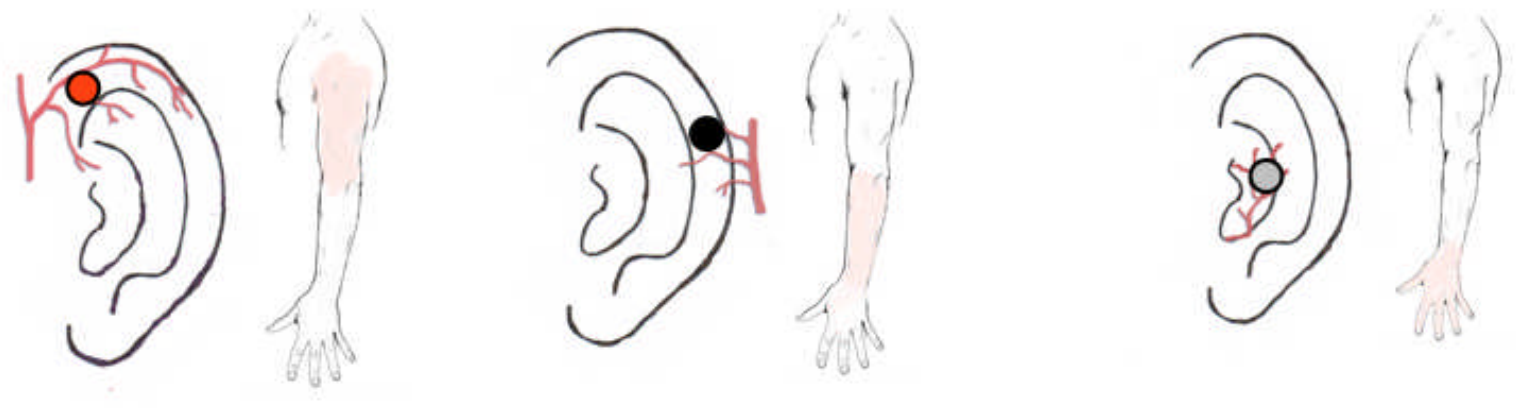

VAS RELATIVE TO PHANTOM LIMB SENSATION

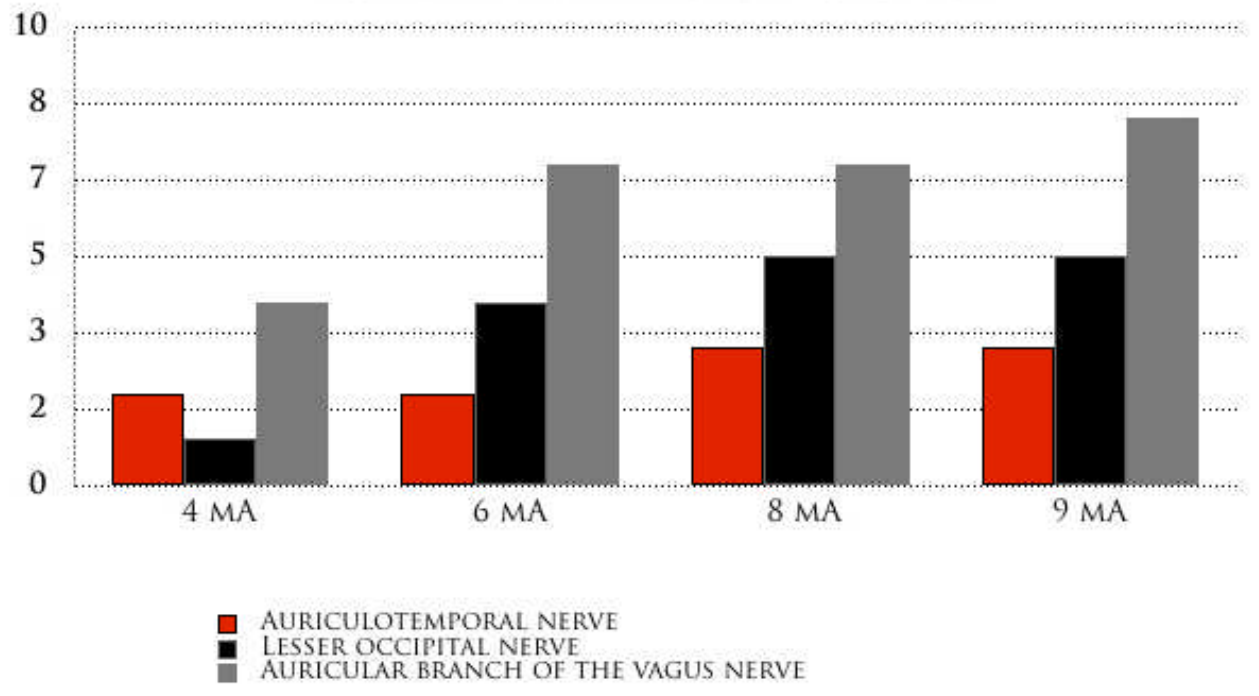




\subsection{SEP recordings}

On the basis of non-painful electrical stimulation, zone 4 (see Fig. 3) of both ears was explored by the time-course of the SEPs. Visual inspection of waveforms and maps revealed negative-positive peak sequences, which emerged in the frontal-central and central-parietal sites, respectively, at about $24 \mathrm{~ms}, 40 \mathrm{~ms}$, and $66 \mathrm{~ms}$ (N-P20; N-P40; N-P60) after stimulation of both ears. These peaks appeared larger after left ear stimulation than after right ear stimulation (Fig. 5). To examine the critical peaks at which differences in amplitudes between the left and right ears would emerge, we employed a non-parametric bootstrap technique (Efron, 2000).

Based on the scalp topography, we selected the electrodes where the negative and positive peaks were maximal and averaged them to obtain a frontal-central and a central-parietal pool. The frontalcentral pool included F1/2 and F3/4; FC1/2, FC3/4, F1/2 and F3/4; and FCz and FC1/2 respectively for N24, N40, and N66 peaks for right and left ear stimulations. The central-parietal pool included CP1/2, CP3/4; P5/6 and CP5/6; and P5/6 and P7/8 respectively for P24, P40, and P66 peaks for right and left ear stimulations.

For the statistical analysis, the amplitude of each component for the left and right ears was estimated by calculating the average of the evoked potential within a time period of $7 \mathrm{~ms}$ centered on the maximal peak of each component. The difference between the mean peaks in these temporal windows was taken to represent the left-right ear contrast. Then we formed two new sets of randomly assigned EEG trials and calculated the mean amplitude of negative frontal-central and positive central-parietal peaks in the selected time windows. Finally, we computed the difference between the mean amplitude of critical peaks of the two resampled datasets.

The results of the bootstrap analysis, shown in Fig. 5, yielded significant results in the centralparietal P20 and in the frontal-central N40. These two peaks are temporally consistent with the M20 and M40 SEF components (Nihashi et al., 2003; Nihashi et al., 2001) that were hypothesized to be generated in the primary and secondary somatosensory cortex respectively. 


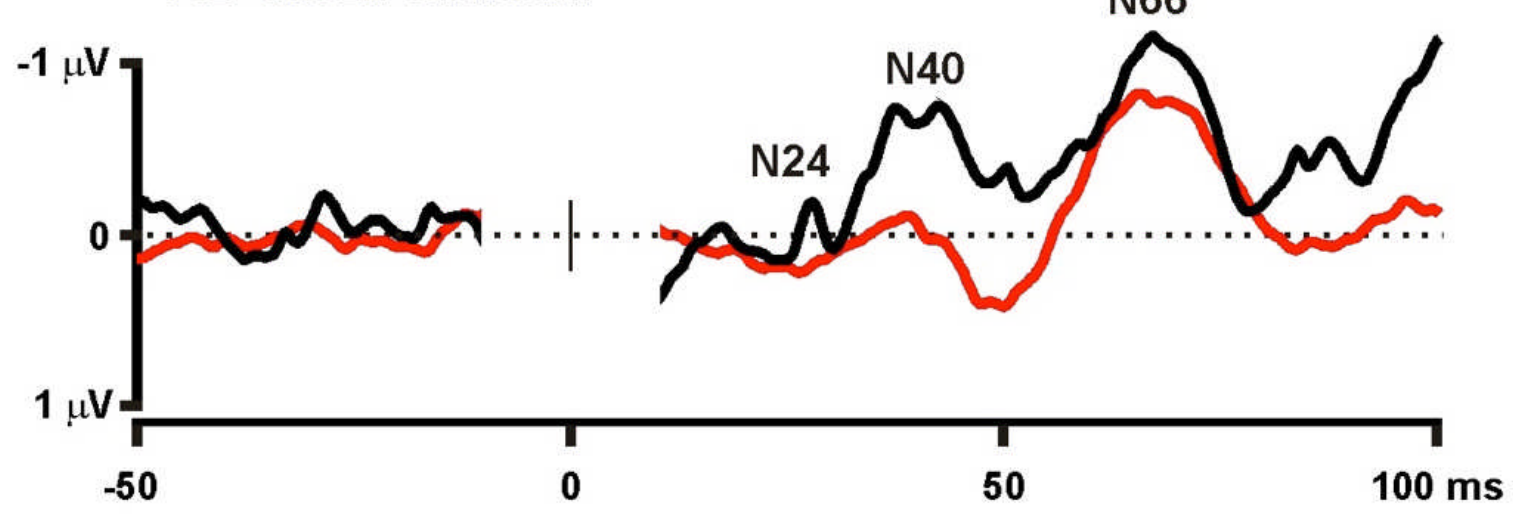

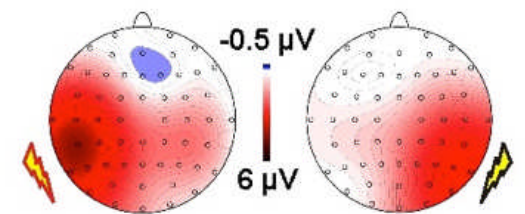

$24 \mathrm{~ms}$

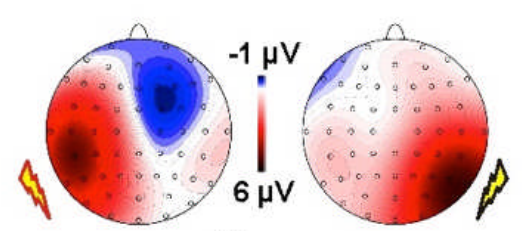

$40 \mathrm{~ms}$

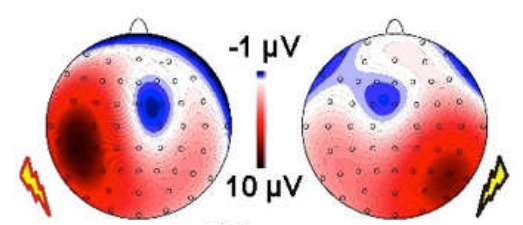

$66 \mathrm{~ms}$
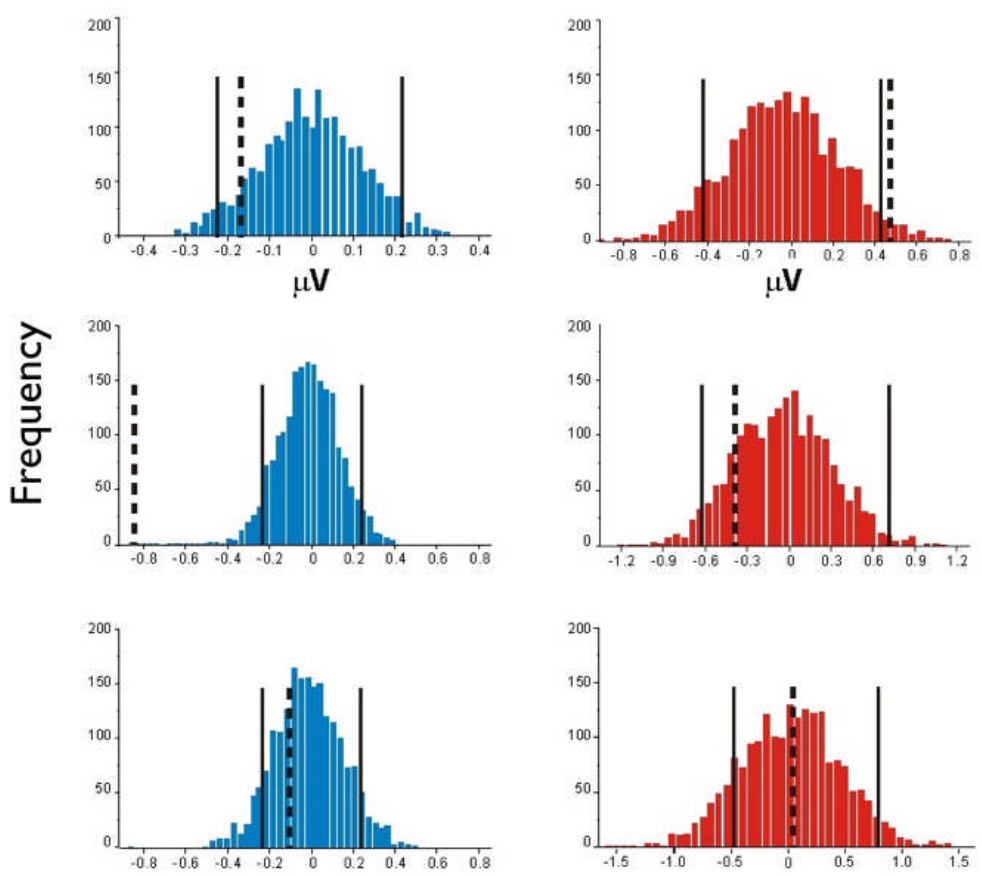

\section{Discussion}

Many somatotopical representations and topographical relationships between body regions and the human brain have been identified (Penfield \& Rasmussen, 1950; Zeharia, Hertz, Flash, \& Amedi, 2015). However, sites activated by ear stimulation have not been clarified, and images of the sensory and motor homunculi do not depict where the ear is, relative to other facial structures. As a matter of fact, the somatosensation of the outer ear has been scarcely studied systematically through the use of modern experimental techniques. According to human studies using noninvasive brain imaging techniques such as functional MRI (Nihashi et al., 2001), somatosensory-evoked magnetic fields (Nihashi et al., 2003), and magnetoencephalography (Nihashi et al., 2002), the representation of the ear in the somatosensory cortex might be located on the border between the neck and face 
(Nihashi et al., 2003; Nihashi et al., 2001; Nihashi et al., 2002) or close to the side and back of the head (Frangos, Ellrich, \& Komisaruk, 2015), with a degree of variability among subjects. Ear movement without movement of other parts of the body can be elicited by electrical cortical stimulation in humans (Yu et al., 2010) and in primates (Bon \& Lucchetti, 1994; Parthasarathy, Schall, \& Graybiel, 1992), suggesting the presence of a specific motor representation of the ear, probably within or close to the eye fields (Bon \& Lucchetti, 1994; Parthasarathy et al., 1992). Therefore, as one would expect from clinical observations of limb amputation (Pourrier et al., 2010) and mastectomy (Aglioti, Cortese, \& Franchini, 1994), phantom sensations can be specific to the ear rather than the face and neck. Here, we report that sensations on one specific manual nerve territory were remapped to ear nerve territories, in the cases of both manual and electrical stimulation. Such phantom experiences appear to reflect a topography-respecting transfer of a spatially organized limb map to the skin of the outer ear. In particular, stimulation of the central internal portion of the outer ear, the concha, yielded sensation arising from the dorsal and volar surface of the left hand, and in a clearly delineated fashion, on the digits extending from the little finger to thumb. The amplitude of SEPs indicated that signals following stimulation of this portion of ear reach both areas of the somatosensory cortex.

SEPs documented that the amplitude of the frontal N40 and parietal P20 components along with corresponding dipolar sources strength were significantly different after stimulation of the two ears. These differences may be related to the reduced activity of a dipolar source in the primary somatosensory cortex. These neurophysiological findings indicate that the primary somatosensory cortical processing after phantom sensation can be altered by both thalamic and/or cortical gating mechanisms (Allison, McCarthy, Wood, \& Jones, 1991; Allison, Wood, McCarthy, \& Spencer, 1991).

Conversely, the stimulation of the anterior lobule of the ear-which is innervated by the greater auricular nerve (Peuker \& Filler, 2002) — did not induce any specific phantom sensation on the hand and arm. Recent evidence in primates suggests that reorganization in the somatosensory cortex takes place as a result of the cuneate nucleus in the brainstem no longer receiving signals from the deafferented limb (Chand \& Jain, 2015; Kambi et al., 2014). It is important to note that the earlobe, which is innervated by the greater auricular nerve, projects to the nucleus cuneatus (Peuker \& Filler, 2002); while the cymba conchae of the external ear, where the patient referred phantom sensation, is innervated exclusively by the sensory auricular branch of the vagus nerve (Peuker \& Filler, 2002). Vagus nerve, which bypass the spinal cord, provide the afferent pathway for the somatosensory cortex, at least when visualized by neuroimaging (Frangos et al., 2015; Komisaruk et al., 2004). Therefore, the ascending contributions of vagus nerve could influence cortical map 
plasticity (Redgrave et al., 2018). Thus, although plasticity in the brainstem likely contributes to phantom sensations in cases of deafferentation, our results suggest that at least part of the neural rearrangement induced by the ear can be genuinely cortical. This finding may help reconcile the discrepancy between the initially proposed functional reorganization and plasticity (Pons et al., 1991), as seen in face-related activity in the missing hand cortex of monkeys (Kaas, Merzenich, \& Killackey, 1983; Merzenich et al., 1984), and recent findings in humans, showing little change of activity in somatosensory cortex with preserved digit somatotopy following amputation (Kikkert et al., 2016). However, these processes are not mutually exclusive, and both might contribute to remapping on face and ear area. Phantom limb sensations in our patient occurred almost immediately after injury, and this suggests that plastic reorganization processes are immediate. It is important to highlight that in two other reported clinical observations, rapid phantom sensation specific to the earlobe rather than the face occurred rapidly, within five days (Aglioti et al., 1994) and into five weeks (Pourrier et al., 2010) after injury. Other studies, however, have identified the transfer of a phantom sensation to the face six months after PBI (Tsao et al., 2016) and 29 years after amputation (Ramachandran, Stewart, \& Rogers-Ramachandran, 1992) . A dual plastic neural process of the somatosensory cortex map thus appears: first to the ear area, and perhaps subsequently to the face area. This raises the possibility that remapping could occur flexibly across multiple time scales (Pascual-Leone, Peris, Tormos, Pascual, \& Catala, 1996), and perhaps move within a short timeframe toward progressive adjustments to neural reorganization. Decreased and increased cortical responsiveness can therefore coexist offering a dual temporal perspective of cortical reorganization after deafferentation (Humanes-Valera, Foffani, Alonso-Calvino, FernandezLopez, \& Aguilar, 2017). Therefore, short and long-term modifications to neurotransmitter concentrations (e.g., acetylcholine and glutamate), which are reversible, have been assessed in deafferented somatosensory areas of primates (Conti, Minelli, \& Pons, 1996). In our patient, as seen shortly after trauma, there was no observable reassignment of the face to the affected limb. Future longitudinal studies could potentially clarify the precise timing of reorganization and referred sensation. The time course of neuroplastic change may drive not only phantom sensations, but also phantom pain. While phantom limb sensations can arise almost immediately during brachial blocks (Gentili, Verton, Kinirons, \& Bonnet, 2002), phantom pain in BPI generally begin six to nine months after injury (Shankar et al., 2015; Sindou, Blondet, Emery, \& Mertens, 2005). Our patient reported that the worst pain was on the physically affected limb, as is commonly seen after an injury to the brachial plexus and not in phantom limb. It remains unclear, even for the pain, whether phantom sensation remains stable in the longer term, or is in continuous flux. For example, the phantom limb might be followed by phantom pain after a longer period of plastic change. Further, 
in our patient, not only was phantom pain absent during stimulation of the ear, but also the physical pain of the affected limb improved. This effect of pain modulation is consistent with outer ear stimulation depending on vagal innervation. The projections of the vague nerve induce activation of locus coeruleus, periaqueductal gray, and dorsal raphe (Frangos et al., 2015). These areas in turn provide descending pain inhibitory pathways to the spinal cord dorsal horn (Millan, 2002).

\section{Conclusion}

The reorganization of the neural connections that define the brain's precise topography should be considered not only a deficit but, under specific circumstances as rehabilitation, a usefully exploitation to evoke sensations within the cortex and to enhance somatic awareness of the denervated body, by stimulating other body parts (Lenggenhager, Scivoletto, Molinari, \& Pazzaglia, 2013). The brain seems to maintain stable bodily representation following deafferentation, and this could serve as an important factor in preventing maladaptive neural plasticity. Our results suggest that referred sensations following brachial plexus injury can evoke the conscious representation of a specific body part in the somatosensory cortex, and that this correspondence remains fixed throughout one's life, independently of changes in the sensory or motor periphery and irrespectively of neuroplasticity effects. Stimulation of the auricular branch of the vagus nerve paired with rehabilitative training improves the perception limb by boosting neuroplasticity (Redgrave et al., 2018).

This opens the possibility of therapies designed to preserve or restore precise cortical topographyeven when sensations are transferred to a different cortical territory-preserving body representation, and potentially restoring sensorimotor function (Lenggenhager, Pazzaglia, Scivoletto, Molinari, \& Aglioti, 2012). Sensory and motor signals arriving from remote body parts, as a result of plasticity, could be valuable in restoring sensorimotor function (Pazzaglia, 2015; Pazzaglia \& Zantedeschi, 2016). This intrinsic neural plasticity could be harnessed to support integration of neuroprosthetics with the human body (Pazzaglia, Galli, Scivoletto, \& Molinari, 2013; Pazzaglia \& Molinari, 2016). Referred sensory effects could also serve as a viable intervention in reducing the perception of pain (Pazzaglia, Haggard, Scivoletto, Molinari, \& Lenggenhager, 2016) or in restoring mental representation of one's own body and the sense of bodily self (Galli, Lenggenhager, Scivoletto, Molinari, \& Pazzaglia, 2015; Galli \& Pazzaglia, 2015) following deafferentation.

\section{Caption to Figures}


Figure 1. Magnetic resonance images reveal nerve root avulsion at the divisions and cords of left C6-C7.

Hypointense (a) and hyperintense (b) signals at the divisions and cords of the left lateral spinal nerve due to hemosiderinic deposits. Extradural and intraforaminal pseudocystis at the T1-T2 vertebral level, with the disappearance of the corresponding root (c). Fibrosis of the plexus lateral to the scalene muscles and the retroclavicular (d).

Figure 2. Schematic demarcation of dermatomes in accordance with Keegan and Garrett showing the distinct points stimulated (Keegan \& Garrett, 1948). The graph shows the \% correct response for 0,1 , and 2 touch trials on each skin region.

Figure 3. A.M.'s referred sensations using manual stimulation of the ear. Note the spatial pattern of referred sensation in the phantom limb, felt when different locations on the face and ear were stimulated. The bottom left diagram delineates the four sensory nerves supplying the skin of the ear.

Figure 4. A.M. referred sensations using non-noxious electrical stimulation. Note the change in intensity of the referred sensation in the phantom limb that was felt when three different locations on the ear were stimulated by 4 different intensities ranging from 4 to $9 \mathrm{~mA}$.

Figure 5. Top panel. Grand-average SEP recordings at two representative electrode sites (FC1 and FC2) for the right (red line) and left (black line) ear stimulations.

Left bottom panel. Scalp topographies of the three investigated SEP components in the two stimulation conditions.

Right bottom panel. Illustration of the bootstrap sampling distributions of the difference in the left-right ears. Histograms of the left-right ear stimulation contrast values obtained from 2000 resampled datasets formed for each peak (from left to right: N-P20; N-P40; N-P60) and each electrode pool (blue bars histogram: frontal-central pool; red bars histogram: central-parietal pool) are referred to as the bootstrap distribution of the statistic. The vertical black lines of the histograms represent the lower and higher 5th percentile points of the distributions and served as the critical values for the (two-tailed) significance at the .05 level. The dashed black lines indicate the left-right ear stimulation contrast observed in our patient. The results of the bootstrap analysis lie in the lower $2.5 \%$ (frontal-central N40) and upper $2.5 \%$ (central-parietal P20) tails, showing that the differences in the left and right ear stimulations of A.M. are reliably different from zero.

\section{References}

Aglioti, S., Cortese, F., \& Franchini, C. (1994). Rapid sensory remapping in the adult human brain as inferred from phantom breast perception. [Comparative Study

Research Support, Non-U.S. Gov't]. Neuroreport, 5(4), 473-476.

Allison, T., McCarthy, G., Wood, C. C., \& Jones, S. J. (1991). Potentials evoked in human and monkey cerebral cortex by stimulation of the median nerve. A review of scalp and intracranial recordings. Brain, 114 ( Pt 6), 2465-2503.

Allison, T., Wood, C. C., McCarthy, G., \& Spencer, D. D. (1991). Cortical somatosensory evoked potentials. II. Effects of excision of somatosensory or motor cortex in humans and monkeys. J Neurophysiol, 66(1), 64-82. doi: 10.1152/jn.1991.66.1.64

Bhat, D. I., Indira Devi, B., Bharti, K., \& Panda, R. (2017). Cortical plasticity after brachial plexus injury and repair: a resting-state functional MRI study. Neurosurg Focus, 42(3), E14. doi: $10.3171 / 2016.12$.FOCUS16430 
Birbaumer, N., Lutzenberger, W., Montoya, P., Larbig, W., Unertl, K., Topfner, S., . . Flor, H. (1997). Effects of regional anesthesia on phantom limb pain are mirrored in changes in cortical reorganization. J Neurosci, 17(14), 5503-5508.

Bon, L., \& Lucchetti, C. (1994). Ear and eye representation in the frontal cortex, area 8b, of the macaque monkey: an electrophysiological study. Exp Brain Res, 102(2), 259-271.

Chand, P., \& Jain, N. (2015). Intracortical and Thalamocortical Connections of the Hand and Face Representations in Somatosensory Area 3b of Macaque Monkeys and Effects of Chronic Spinal Cord Injuries. J Neurosci, 35(39), 13475-13486. doi: 10.1523/JNEUROSCI.2069-15.2015

Collins, K. L., McKean, D. L., Huff, K., Tommerdahl, M., Favorov, O. V., Waters, R. S., \& Tsao, J. W. (2017). Hand-to-Face Remapping But No Differences in Temporal Discrimination Observed on the Intact Hand Following Unilateral Upper Limb Amputation. Front Neurol, 8, 8. doi: 10.3389/fneur.2017.00008

Conti, F., Minelli, A., \& Pons, T. P. (1996). Changes in glutamate immunoreactivity in the somatic sensory cortex of adult monkeys induced by nerve cuts. J Comp Neurol, 368(4), 503-515. doi: 10.1002/(SICI)1096-9861(19960513)368:4\&lt;503::AIDCNE3\&gt;3.0.CO;2-8

Cook, A. W., \& Druckemiller, W. H. (1952). Phantom limb in paraplegic patients; report of two cases and an analysis of its mechanism. J Neurosurg, 9(5), 508-516. doi: 10.3171/jns.1952.9.5.0508

Efron, B. (2000). The bootstrap and modern statistics. J Am Stat Assoc, 95, 1293-1296.

Finnerup, N. B., Norrbrink, C., Fuglsang-Frederiksen, A., Terkelsen, A. J., Hojlund, A. P., \& Jensen, T. S. (2010). Pain, referred sensations, and involuntary muscle movements in brachial plexus injury. [Research Support, Non-U.S. Gov't]. Acta Neurol Scand, 121(5), 320-327. doi: 10.1111/j.1600-0404.2009.01248.x

Flor, H., Elbert, T., Knecht, S., Wienbruch, C., Pantev, C., Birbaumer, N., . . Taub, E. (1995). Phantom-limb pain as a perceptual correlate of cortical reorganization following arm amputation. Nature, 375(6531), 482-484. doi: 10.1038/375482a0

Flor, H., Nikolajsen, L., \& Staehelin Jensen, T. (2006). Phantom limb pain: a case of maladaptive CNS plasticity? [Research Support, Non-U.S. Gov't

Review]. Nat Rev Neurosci, 7(11), 873-881. doi: 10.1038/nrn1991

Frangos, E., Ellrich, J., \& Komisaruk, B. R. (2015). Non-invasive Access to the Vagus Nerve Central Projections via Electrical Stimulation of the External Ear: fMRI Evidence in Humans. Brain Stimul, 8(3), 624-636. doi: 10.1016/j.brs.2014.11.018

Galli, G., Lenggenhager, B., Scivoletto, G., Molinari, M., \& Pazzaglia, M. (2015). Don't look at my wheelchair! The plasticity of longlasting prejudice. Med Educ, 49(12), 1239-1247. doi: 10.1111/medu.12834

Galli, G., \& Pazzaglia, M. (2015). Commentary on: "The body social: an enactive approach to the self". A tool for merging bodily and social self in immobile individuals. Front Psychol, 6, 305. doi: 10.3389/fpsyg.2015.00305

Gentili, M. E., Verton, C., Kinirons, B., \& Bonnet, F. (2002). Clinical perception of phantom limb sensation in patients with brachial plexus block. Eur J Anaesthesiol, 19(2), 105-108.

Haenzi, S., Stefanics, G., Lanaras, T., Calcagni, M., \& Ghosh, A. (2014). Altered cortical activation from the hand after facial botulinum toxin treatment. Ann Clin Transl Neurol, 1(1), 6468. doi: $10.1002 / \operatorname{acn} 3.21$

Halligan, P. W., Marshall, J. C., \& Wade, D. T. (1994). Sensory disorganization and perceptual plasticity after limb amputation: a follow-up study. Neuroreport, 5(11), 1341-1345.

Humanes-Valera, D., Foffani, G., Alonso-Calvino, E., Fernandez-Lopez, E., \& Aguilar, J. (2017). Dual Cortical Plasticity After Spinal Cord Injury. Cereb Cortex, 27(5), 2926-2940. doi: 10.1093/cercor/bhw142 
Hunter, J. P., Katz, J., \& Davis, K. D. (2003). The effect of tactile and visual sensory inputs on phantom limb awareness. [Research Support, Non-U.S. Gov't]. Brain, 126(Pt 3), 579589.

Ichinose, A., Sano, Y., Osumi, M., Sumitani, M., Kumagaya, S. I., \& Kuniyoshi, Y. (2017). Somatosensory Feedback to the Cheek During Virtual Visual Feedback Therapy Enhances Pain Alleviation for Phantom Arms. Neurorehabil Neural Repair, 31(8), 717725. doi: $10.1177 / 1545968317718268$

Kaas, J. H., Merzenich, M. M., \& Killackey, H. P. (1983). The reorganization of somatosensory cortex following peripheral nerve damage in adult and developing mammals. Annu Rev Neurosci, 6, 325-356. doi: 10.1146/annurev.ne.06.030183.001545

Kambi, N., Halder, P., Rajan, R., Arora, V., Chand, P., Arora, M., \& Jain, N. (2014). Large-scale reorganization of the somatosensory cortex following spinal cord injuries is due to brainstem plasticity. Nat Commun, 5, 3602. doi: 10.1038/ncomms4602

Katz, J. (1992). Psychophysiological contributions to phantom limbs. Can J Psychiatry, 37(5), 282-298.

Keegan, J. J., \& Garrett, F. D. (1948). The segmental distribution of the cutaneous nerves in the limbs of man. Anat Rec., 102, 409-437.

Kikkert, S., Kolasinski, J., Jbabdi, S., Tracey, I., Beckmann, C. F., Johansen-Berg, H., \& Makin, T. R. (2016). Revealing the neural fingerprints of a missing hand. Elife, 5. doi: 10.7554/eLife.15292

Komisaruk, B. R., Whipple, B., Crawford, A., Liu, W. C., Kalnin, A., \& Mosier, K. (2004). Brain activation during vaginocervical self-stimulation and orgasm in women with complete spinal cord injury: fMRI evidence of mediation by the vagus nerves. Brain Res, 1024(12), 77-88. doi: 10.1016/j.brainres.2004.07.029

Lenggenhager, B., Pazzaglia, M., Scivoletto, G., Molinari, M., \& Aglioti, S. M. (2012). The sense of the body in individuals with spinal cord injury. PLoS One, 7(11), e50757. doi: 10.1371/journal.pone.0050757

Lenggenhager, B., Scivoletto, G., Molinari, M., \& Pazzaglia, M. (2013). Restoring tactile awareness through the rubber hand illusion in cervical spinal cord injury. Neurorehabil Neural Repair, 27(8), 704-708. doi: 10.1177/1545968313491009

Lotze, M., Flor, H., Grodd, W., Larbig, W., \& Birbaumer, N. (2001). Phantom movements and pain. An fMRI study in upper limb amputees. Brain, 124(Pt 11), 2268-2277.

Makin, T. R., \& Bensmaia, S. J. (2017). Stability of Sensory Topographies in Adult Cortex. Trends Cogn Sci, 21(3), 195-204. doi: 10.1016/j.tics.2017.01.002

Makin, T. R., Scholz, J., Filippini, N., Henderson Slater, D., Tracey, I., \& Johansen-Berg, H. (2013). Phantom pain is associated with preserved structure and function in the former hand area. Nat Commun, 4, 1570. doi: 10.1038/ncomms2571

Makin, T. R., Scholz, J., Henderson Slater, D., Johansen-Berg, H., \& Tracey, I. (2015). Reassessing cortical reorganization in the primary sensorimotor cortex following arm amputation. Brain, 138(Pt 8), 2140-2146. doi: 10.1093/brain/awv161

Melzack, R. (1990). Phantom limbs and the concept of a neuromatrix. Trends Neurosci, 13(3), 88-92.

Merzenich, M. M., Nelson, R. J., Stryker, M. P., Cynader, M. S., Schoppmann, A., \& Zook, J. M. (1984). Somatosensory cortical map changes following digit amputation in adult monkeys. J Comp Neurol, 224(4), 591-605. doi: 10.1002/cne.902240408

Millan, M. J. (2002). Descending control of pain. Prog Neurobiol, 66(6), 355-474.

Montoya, P., Ritter, K., Huse, E., Larbig, W., Braun, C., Topfner, S., ... Birbaumer, N. (1998). The cortical somatotopic map and phantom phenomena in subjects with congenital limb atrophy and traumatic amputees with phantom limb pain. Eur J Neurosci, 10(3), 10951102. 
Nihashi, T., Kakigi, R., Hoshiyama, M., Miki, K., Kajita, Y., Yoshida, J., \& Yatsuya, H. (2003). Effect of tactile interference stimulation of the ear in human primary somatosensory cortex: a magnetoencephalographic study. Clin Neurophysiol, 114(10), 1866-1878.

Nihashi, T., Kakigi, R., Kawakami, O., Hoshiyama, M., Itomi, K., Nakanishi, H., . . Yoshida, J. (2001). Representation of the ear in human primary somatosensory cortex. Neuroimage, 13(2), 295-304. doi: 10.1006/nimg.2000.0695

Nihashi, T., Kakigi, R., Okada, T., Sadato, N., Kashikura, K., Kajita, Y., \& Yoshida, J. (2002). Functional magnetic resonance imaging evidence for a representation of the ear in human primary somatosensory cortex: comparison with magnetoencephalography study. Neuroimage, 17(3), 1217-1226.

Parry, C. B. (1980). Pain in avulsion lesions of the brachial plexus. Pain, 9(1), 41-53.

Parthasarathy, H. B., Schall, J. D., \& Graybiel, A. M. (1992). Distributed but convergent ordering of corticostriatal projections: analysis of the frontal eye field and the supplementary eye field in the macaque monkey. J Neurosci, 12(11), 4468-4488.

Pascual-Leone, A., Peris, M., Tormos, J. M., Pascual, A. P., \& Catala, M. D. (1996). Reorganization of human cortical motor output maps following traumatic forearm amputation. Neuroreport, 7(13), 2068-2070.

Pazzaglia, M. (2015). Body and Odors: Non Just Molecules, After All. Current directions in psychological science, 24(4), 329-333.

Pazzaglia, M., Galli, G., Scivoletto, G., \& Molinari, M. (2013). A functionally relevant tool for the body following spinal cord injury. [Research Support, Non-U.S. Gov't]. PLoS One, 8(3), e58312. doi: 10.1371/journal.pone.0058312

Pazzaglia, M., Haggard, P., Scivoletto, G., Molinari, M., \& Lenggenhager, B. (2016). Pain and somatic sensation are transiently normalized by illusory body ownership in a patient with spinal cord injury. Restor Neurol Neurosci, 34(4), 603-613. doi: 10.3233/RNN150611

Pazzaglia, M., \& Molinari, M. (2016). The embodiment of assistive devices-from wheelchair to exoskeleton. Phys Life Rev, 16, 163-175. doi: 10.1016/j.plrev.2015.11.006

Pazzaglia, M., \& Zantedeschi, M. (2016). Plasticity and awareness of bodily distortion. Neural Plasticity.

Penfield, W., \& Rasmussen, T. (1950). The cerebral cortex of man. The Macmillan Company, New York.

Peuker, E. T., \& Filler, T. J. (2002). The nerve supply of the human auricle. Clin Anat, 15(1), 3537. doi: $10.1002 /$ ca.1089

Pons, T. P., Garraghty, P. E., Ommaya, A. K., Kaas, J. H., Taub, E., \& Mishkin, M. (1991). Massive cortical reorganization after sensory deafferentation in adult macaques. Science, 252(5014), 1857-1860.

Pourrier, S. D., Nieuwstraten, W., Van Cranenburgh, B., Schreuders, T. A., Stam, H. J., \& Selles, R. W. (2010). Three cases of referred sensation in traumatic nerve injury of the hand: implications for understanding central nervous system reorganization. J Rehabil Med, 42(4), 357-361. doi: 10.2340/16501977-0526

Ramachandran, V. S., \& Hirstein, W. (1998). The perception of phantom limbs. The D. O. Hebb lecture. Brain, 121 ( Pt 9), 1603-1630.

Ramachandran, V. S., \& Rogers-Ramachandran, D. (2000). Phantom limbs and neural plasticity. Arch Neurol, 57(3), 317-320.

Ramachandran, V. S., Stewart, M., \& Rogers-Ramachandran, D. C. (1992). Perceptual correlates of massive cortical reorganization. Neuroreport, 3(7), 583-586.

Redgrave, J. N., Moore, L., Oyekunle, T., Ebrahim, M., Falidas, K., Snowdon, N., . . Majid, A. (2018). Transcutaneous Auricular Vagus Nerve Stimulation with Concurrent Upper 
Limb Repetitive Task Practice for Poststroke Motor Recovery: A Pilot Study. J Stroke Cerebrovasc Dis, 27(7), 1998-2005. doi: 10.1016/j.jstrokecerebrovasdis.2018.02.056

Shankar, H., Hansen, J., \& Thomas, K. (2015). Phantom pain in a patient with brachial plexus avulsion injury. Pain Med, 16(4), 777-781. doi: 10.1111/pme.12635

Sherman, R. A., Griffin, V. D., Evans, C. B., \& Grana, A. S. (1992). Temporal relationships between changes in phantom limb pain intensity and changes in surface electromyogram of the residual limb. Int J Psychophysiol, 13(1), 71-77.

Sindou, M. P., Blondet, E., Emery, E., \& Mertens, P. (2005). Microsurgical lesioning in the dorsal root entry zone for pain due to brachial plexus avulsion: a prospective series of 55 patients. J Neurosurg, 102(6), 1018-1028. doi: 10.3171/jns.2005.102.6.1018

Son, B. C., \& Ha, S. W. (2015). Phantom Remodeling Effect of Dorsal Root Entry Zone Lesioning in Phantom Limb Pain Caused by Brachial Plexus Avulsion. Stereotact Funct Neurosurg, 93(4), 240-244. doi: 10.1159/000381556

Tsao, J. W., Finn, S. B., \& Miller, M. E. (2016). Reversal of phantom pain and hand-to-face remapping after brachial plexus avulsion. Ann Clin Transl Neurol, 3(6), 463-464. doi: 10.1002/acn3.316

Weeks, S. R., \& Tsao, J. W. (2010). Incorporation of another person's limb into body image relieves phantom limb pain: A case study. Neurocase, 16(6), 461-465. doi: Pii 921668417

$10.1080 / 13554791003730592$

Yu, L., Terada, K., Usui, N., Usui, K., Baba, K., \& Inoue, Y. (2010). Ear movement induced by electrical cortical stimulation. Epilepsy Behav, 18(4), 488-490. doi: 10.1016/j.yebeh.2010.05.016

Zeharia, N., Hertz, U., Flash, T., \& Amedi, A. (2015). New whole-body sensory-motor gradients revealed using phase-locked analysis and verified using multivoxel pattern analysis and functional connectivity. J Neurosci, 35(7), 2845-2859. doi: 10.1523/JNEUROSCI.4246-14.2015 